\title{
Swimming Propulsion Forces Are Enhanced by a Small Finger Spread
}

\section{Daniel A. Marinho, Tiago M. Barbosa, Victor M. Reis, Per L. Kjendlie, Francisco B. Alves, João P. Vilas-Boas, Leandro Machado, António J. Silva, and Abel I. Rouboa}

\begin{abstract}
The main aim of this study was to investigate the effect of finger spread on the propulsive force production in swimming using computational fluid dynamics. Computer tomography scans of an Olympic swimmer hand were conducted. This procedure involved three models of the hand with differing finger spreads: fingers closed together (no spread), fingers with a small $(0.32 \mathrm{~cm})$ spread, and fingers with large $(0.64 \mathrm{~cm})$ spread. Steady-state computational fluid dynamics analyses were performed using the Fluent code. The measured forces on the hand models were decomposed into drag and lift coefficients. For hand models, angles of attack of $0^{\circ}, 15^{\circ}, 30^{\circ}, 45^{\circ}, 60^{\circ}, 75^{\circ}$, and $90^{\circ}$, with a sweep back angle of $0^{\circ}$, were used for the calculations. The results showed that the model with a small spread between fingers presented higher values of drag coefficient than did the models with fingers closed and fingers with a large spread. One can note that the drag coefficient presented the highest values for an attack angle of $90^{\circ}$ in the three hand models. The lift coefficient resembled a sinusoidal curve across the attack angle. The values for the lift coefficient presented few differences among the three models, for a given attack angle. These results suggested that fingers slightly spread could allow the hand to create more propulsive force during swimming.
\end{abstract}

Keywords: hand shape, numerical simulations, computational fluid dynamics, forces, competitive swimming

The study of human swimming propulsion is one of the most complex areas of interest in sport biomechanics (Payton et al., 2002). Over the past decades, research in swimming biomechanics has evolved from the observation of a subject's kinematics to a basic flow dynamics approach, following the line of the scientists working on

Marinho is with the Department of Sport Sciences, University of Beira Interior, Covilhã, Portugal, and the Centre of Research in Sports, Health, and Human Development, Vila Real, Portugal. Barbosa is with the Centre of Research in Sports, Health, and Human Development, Vila Real, Portugal, and the Polytechnic Institute of Bragança, Bragança, Portugal. Reis is with the Centre of Research in Sports, Health, and Human Development, Vila Real, Portugal, and the University of Trás-os-Montes and Alto Douro, Vila Real, Portugal. Kjendlie is with the Norwegian School of Sport Sciences, Oslo, Norway. Alves is with the Faculty of Human Kinetics, Technical University of Lisbon, Lisbon, Portugal. Vilas-Boas is with the Faculty of Sport, University of Porto, Porto, Portugal. Machado is with the Faculty of Sport, University of Porto, Porto, Portugal. Silva is with the Centre of Research in Sports, Health, and Human Development, Vila Real, Portugal, and the University of Trás-os-Montes and Alto Douro, Vila Real, Portugal. Rouboa is with the University of Trás-os-Montes and Alto Douro, Vila Real, Portugal, and the Department of Mechanical Engineering and Applied Mechanics, University of Pennsylvania, Philadelphia, PA. this subject in experimental biology (Dickinson, 2000; Arellano et al., 2006).

Computational fluid dynamics (CFD) is one of the recent methodologies used to achieve this goal. This methodology allows us to analyze the water flow around the human body, to understand the magnitude of drag forces resisting forward motion (Silva et al., 2008; Marinho et al., 2009), and to compute the propulsive forces produced by the propelling segments (Bixler \& Riewald, 2002; Lecrivain et al., 2008).

Computational fluid dynamics could help coaches, in the short term, with technique prescription. Moreover, this methodology could provide answers to some practical issues that remain controversial. The finger's relative position during the underwater path of the stroke cycle is one of these cases. A large intersubject variety of relative finger positioning can be observed during training and competition. Some swimmers (i) maintain the fingers closed together (not spread apart), (ii) others have a small distance between fingers, and (iii) still others have a large distance between fingers. Indeed, the propulsive repercussions of those three possibilities remain unclear for swimming coaches and scientists. There is a lack of research on this issue, and some ideas are passed among members of the swimming community with little empirical (experimental or numerical data) support. Experimental data are controversial: for example, Schleihauf (1979) showed that the fingers closed together and the 
thumb partially abducted allow higher propulsion and Berger (1996) concluded that finger spreading does not influence propulsion. But a more recent paper suggests that fingers closed together induces less propulsion than fingers spread (Sidelnik \& Young, 2006). To our knowledge, there is no research published using a numerical approach on the effect of finger spreading and with anthropometrical data of elite swimmers hands.

Therefore, the main aim of this study was to investigate the effect of finger spread on propulsive force production in swimming using CFD.

\section{Methods}

\section{Three-Dimensional Model}

Scanning. To obtain the geometry of the hand, eight cross-sectional scans of the right hand of an elite swimmer (Figure 1) were conducted using a Toshiba Aquilion 4 computer tomography scanner. Computer tomography scans were obtained with configuration of V2.04 ER001. A 2-mm-slice thickness with a space of $1 \mathrm{~mm}$ was used. The subject was an Olympics-level swimmer who participated in the 2004 Olympic Games, in Athens. The subject was lying prone, with his right arm extended ahead and fully pronated. This procedure was conducted with different finger spreads: fingers closed together, fingers with a small spread (an intrafinger distance of $0.32 \mathrm{~cm}$, from fingertip to fingertip), and fingers with a large spread $(0.64 \mathrm{~cm}$, from fingertip to fingertip) (Schleihauf, 1979). This protocol has been approved by the appropriate ethical committee of the institution in which it was performed and the subject gave informed consent to participate in this work.

Data Manipulation. The transformation of values from the computer tomography scans into nodal coordinates in an appropriate coordinate system warrants the use of image-processing techniques. The image-processing program used in this study was the Anatomics Pro (Anatomics, Saint Kilda, VIC, Australia). This program allowed us to obtain the boundaries of the human segments, creating a three-dimensional reconstruction of the hand. At first, before processing and converting procedures, the data were prepared by observing the computer tomography data and erasing the irrelevant parts of the anatomical model. This step was also conducted using the software FreeForm (SensAble Technologies, Woburn, MA, USA). Finally, the data were converted into an IGES format (*.igs), which could be read by Gambit/Fluent software (Fluent Inc, Lebanon, NH, USA) to define the finite elements approach through the threedimensional surfaces (Figure 2).

\section{CFD Study}

The Fluent code solves flow problems by replacing the Navier-Stokes equations with discretized algebraic expressions that can be solved by iterative computerized

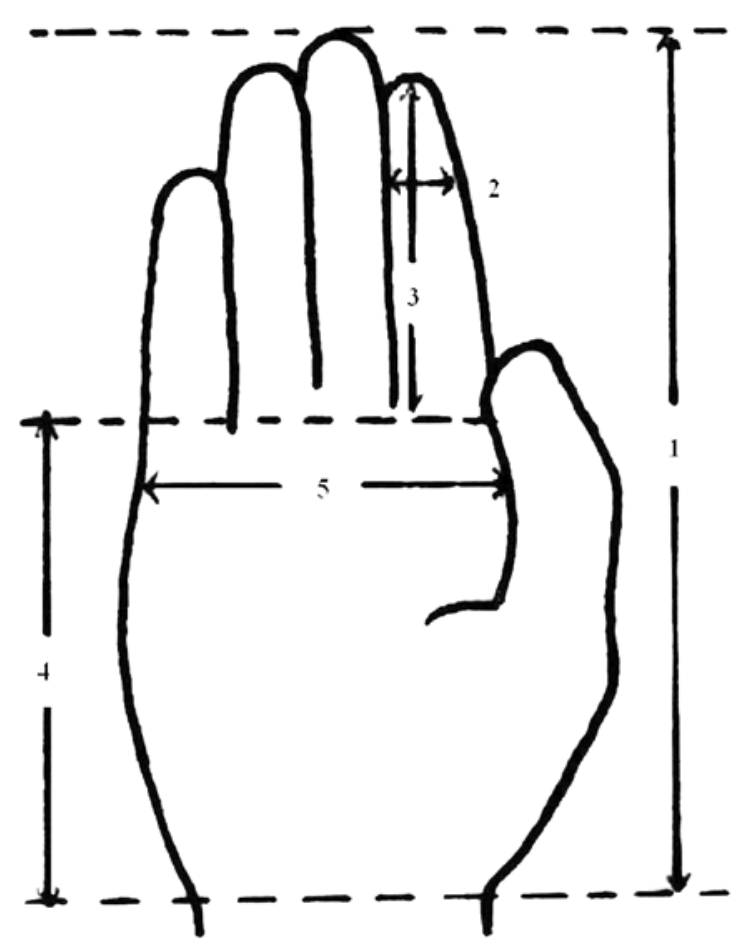

Figure 1 - Anthropometric characteristics of the swimmer's hand. Hand length (1): $20.20 \mathrm{~cm}$, index breadth (2): $1.50 \mathrm{~cm}$, index length (3): $8.10 \mathrm{~cm}$, palm length (4): $9.50 \mathrm{~cm}$, and hand breadth (5): $8.90 \mathrm{~cm}$. 


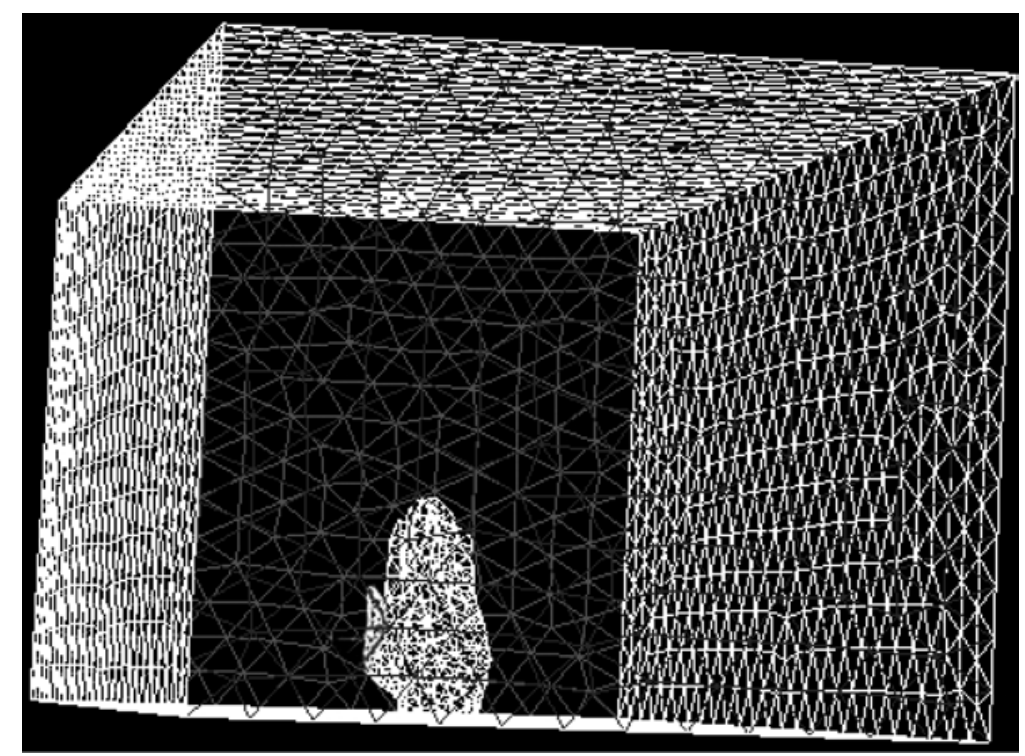

Figure 2 - Computational fluid dynamics model geometry with the hand inside the domain (the model with fingers closed).

calculations. Fluent uses the finite volume approach, where the equations are integrated over each control volume.

The dynamic fluid forces produced by the hand, lift (L) and drag (D), were measured in this study. These forces are functions of the fluid velocity and they were measured by the application of the Equations 1 and 2, respectively:

$$
\begin{aligned}
& \mathrm{D}=\mathrm{C}_{\mathrm{D}} 1 / 2 \rho \mathrm{A \textrm {v } ^ { 2 }} \\
& \mathrm{L}=\mathrm{C}_{\mathrm{L}} 1 / 2 \rho \mathrm{A} \mathrm{v}^{2}
\end{aligned}
$$

In Equations 1 and 2, $v$ is the fluid velocity, $C_{D}$ and $C_{L}$ are the drag and lift coefficients, respectively, $\rho$ is the fluid density, and $\mathrm{A}$ is the projection area of the model for the angles of attack used in this study.

Preprocessing. The whole domain was meshed with a hybrid mesh composed of prisms and pyramids. Significant efforts were conducted to ensure that the model would provide accurate results by decreasing the grid node separation in areas of high velocity and pressure gradients.

Solving Steady Flow. For the calculations, hand model angles of attack of $0^{\circ}, 15^{\circ}, 30^{\circ}, 45^{\circ}, 60^{\circ}, 75^{\circ}$, and $90^{\circ}$, with a sweep back angle of $0^{\circ}$ (thumb as the leading edge) were used (Schleihauf, 1979). Steady-state CFD analyses were performed using the Fluent code, and the drag and lift coefficients were calculated for a flow velocity of $2.0 \mathrm{~m} \cdot \mathrm{s}^{-1}$ (Lauder et al., 2001; Rouboa et al., 2006). We used the segregated solver with the standard K-epsilon turbulence model because this turbulence model was shown to be accurate with measured values in previous research (Moreira et al., 2006).
All numerical computational schemes were second order, which provides a more accurate solution than firstorder schemes. We used a turbulence intensity of $1.0 \%$ and a turbulence scale of $0.10 \mathrm{~m}$. The water temperature was $28{ }^{\circ} \mathrm{C}$ with a density of $998.2 \mathrm{~kg} \cdot \mathrm{m}^{-3}$ and a viscosity of $0.001 \mathrm{~kg} \cdot(\mathrm{m} \cdot \mathrm{s})^{-1}$. Incompressible flow was assumed. The measured forces on the hand models were decomposed into drag $\left(\mathrm{C}_{\mathrm{D}}\right)$ and lift $\left(\mathrm{C}_{\mathrm{L}}\right)$ coefficients, using Equations 1 and 2.

\section{Results}

Figures 3 and 4 show the values of $C_{D}$ and $C_{L}$, respectively, obtained for the hand model with different finger spreads.

One can note that the $\mathrm{C}_{\mathrm{D}}$ presented the highest values for an attack angle of $90^{\circ}$ in the three hand models $(\approx 0.90$ $\left.<\mathrm{C}_{\mathrm{D}}<1.10\right)$. In the three models, the $\mathrm{C}_{\mathrm{D}}$ increased with the attack angle. Moreover, it was possible to observe that for attack angles greater than $30^{\circ}$, the model with the small distance between fingers presented higher values of $\mathrm{C}_{\mathrm{D}}$ when compared with the models with fingers closed and with large finger spread. This last model presented the lowest values of $\mathrm{C}_{\mathrm{D}}$. For attack angles of $0^{\circ}, 15^{\circ}$, and $30^{\circ}$, the values of $C_{D}$ were very similar in the three models of the swimmer's hand.

The $\mathrm{C}_{\mathrm{L}}$ resembled a sinusoidal curve across the attack angle. Maximum values for any hand model occurred near $30^{\circ}-45^{\circ}\left(\mathrm{C}_{\mathrm{L}} \approx 0.60\right)$. Furthermore, the $\mathrm{C}_{\mathrm{L}}$ seemed to be independent of the finger spreading, thus presenting little differences among the three models. However, it was possible to note slightly lower values for the position with a larger distance between fingers, especially for attack angles ranging from $15^{\circ}$ to $60^{\circ}$. 


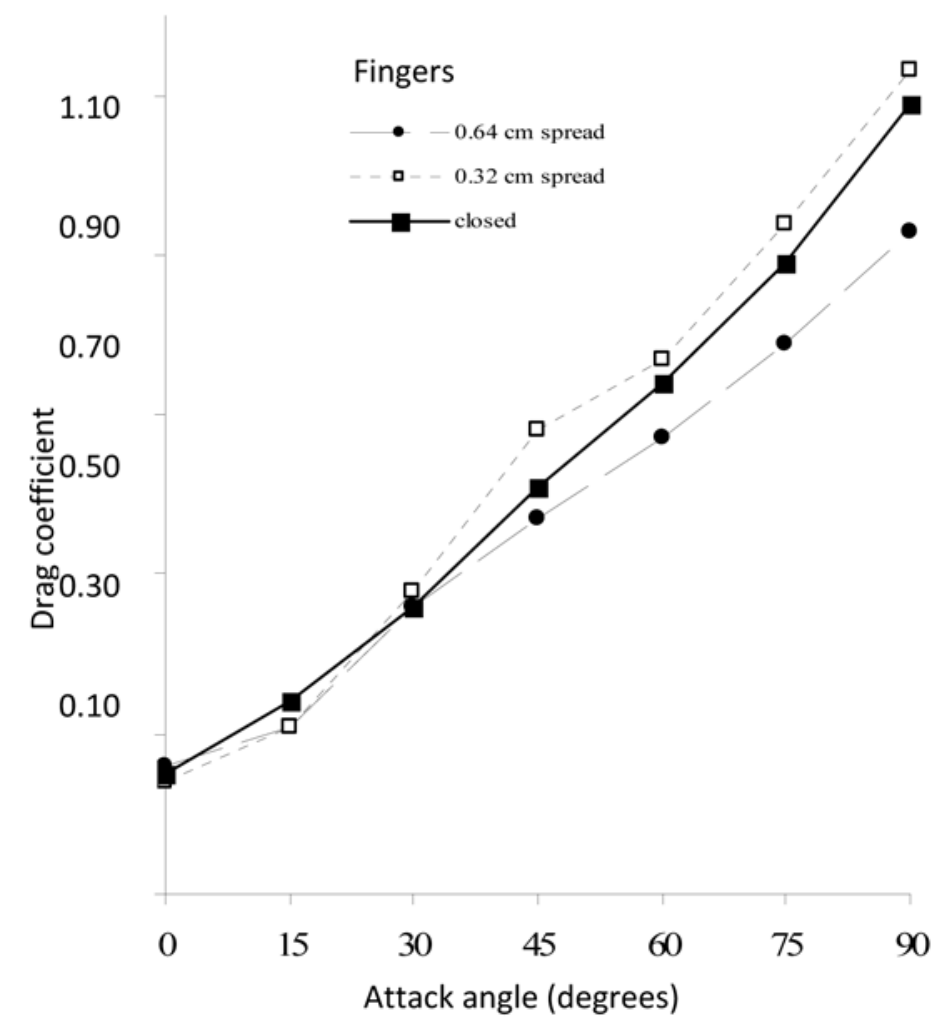

Figure 3 - Values of $\mathrm{C}_{\mathrm{D}}$ obtained for the different attack angles and for the different finger spreads. Sweepback angle $=0^{\circ}$ and flow velocity $=2.0 \mathrm{~m} / \mathrm{s}$.

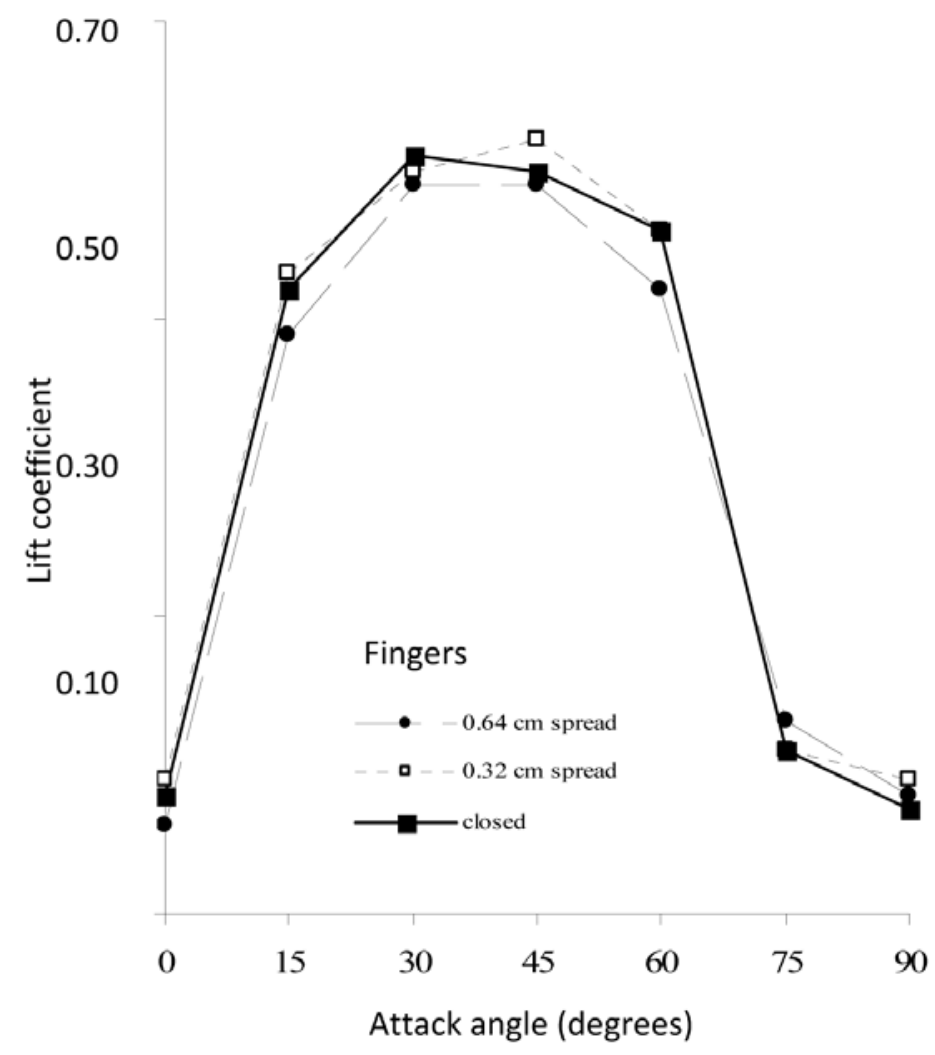

Figure 4 - Values of $\mathrm{C}_{\mathrm{L}}$ obtained for the different attack angles and for the different finger spreads. Sweepback angle $=0^{\circ}$ and flow velocity $=2.0 \mathrm{~m} / \mathrm{s}$. 


\section{Discussion}

The main aim of this study was to analyze the effect of finger spread in the swimming propulsive force production, through CFD. Results suggested that fingers slightly spread could allow the hand to create more propulsive force during swimming.

In this study, we tried to clarify one technical concern of the swimming community: which should be the best finger position to improve force production by the hand during swimming? Therefore, three models with differing finger spread were chosen for the analysis, addressed to characterize different swimming strategies. In addition, the option to analyze one position with fingers closed, one with a small distance between fingers, and another with a large distance between fingers was based on the pioneering study of Schleihauf (1979). Despite some theoretical assumptions and expert opinions (e.g., Counsilman, 1968; Colwin, 1992; Maglischo, 2003), there are few experimental studies to clarify this issue (Schleihauf, 1979; Takagi et al., 2001; Berger, 1996; Sidelnik \& Young, 2006). Rather than an experimental analysis, the current study applied the numerical techniques of CFD to compute the forces produced by the model of the swimmer's hand. Bixler et al. (2007) has already demonstrated the validity of CFD analysis as a tool to examine the water flow around a swimmer's body. Nevertheless, it is very important that the digital model corresponds to a truthful representation of the human segment to ensure accurate numerical results (Candalai \& Reddy, 1992; Lecrivain et al., 2008). Indeed, the computer tomography scans allowed the creation of a true digital model of the swimmer's hand (Aritan et al., 1997). Moreover, precise images of complex 3-D shapes, such as a human hand, obtained by imagiography are becoming widely used in reverse engineering (Lecrivain et al., 2008).

The main finding of the present research was that the model with the small distance between fingers presented higher values of $C_{D}$ than the models with fingers closed together and with fingers spread widely. Furthermore, the $\mathrm{C}_{\mathrm{L}}$ seemed to be independent of the finger spread, presenting few differences among the three models. These results suggest that the use of a position with a small distance between fingers seems to be gainful for swimmers.

The hand position with the small distance between fingers seemed to increase the projection area of the hand, thus increasing force production. The distance between fingers seemed not enough to allow the water to flow freely. Indeed, a turbulent flow between the fingers may be formed, creating some kind of barrier. Nevertheless, regarding the $C_{L}$, the values for the position with the small finger spread and for the position with fingers closed were very similar. For attack angles lower than $90^{\circ}$, the flow above the dorsal surface of the hand, flowing at high velocities, could prevent the flow between fingers. In this condition, assuming that the higher velocity difference between the two surfaces of the swimmers' hand will occur at the attack angle corresponding to the higher $\mathrm{C}_{\mathrm{L}}$ (in this case, between $30^{\circ}$ and $45^{\circ}$ ), it will thus be expected that the so-called barrier will be stronger at those $C_{L}$ values. As can be seen from Figures 3 and 4 , at $\alpha=45^{\circ}$ a relative increase of the $C_{D}$ value is perceptible. This curve tendency corresponds to the maximal $\mathrm{C}_{\mathrm{L}}$ value obtained for the slight spreading condition, and for all studied conditions, indicating the higher flow velocity difference between both faces of the hand. Concerning this, Ungerechts \& Klauck (2006) did suggest having fingers slightly spread to induce flow around the hand at the beginning of the arm cycle.

However, this gain did not occur when we analyzed the greater distance between fingers. In both $\mathrm{C}_{\mathrm{D}}$ and $\mathrm{C}_{\mathrm{L}}$ coefficients, for the position with large finger spread, the values were lower when compared with the positions of fingers closed and slightly spread. For the $\mathrm{C}_{\mathrm{D}}$ and for attack angles higher than $30^{\circ}$, the position with more distance between fingers presented lower values. This position presented also lower values in $\mathrm{C}_{\mathrm{L}}$. It seems that there is a critical distance between fingers beyond which the force production became compromised.

Schleihauf (1979) has already reported an identical situation. The $C_{D}$ for the fingers closed and slightly spread positions presented higher values than the large spread position. In contrast, the values of $C_{L}$ increased in indirect proportion to finger spread for attack angles ranging between $0^{\circ}$ and $60^{\circ}$. Berger (1996) reported that spreading the fingers did not influence propulsive force. Moreover, lift force at attack angles between $60^{\circ}$ and $80^{\circ}$ was higher when spreading the fingers (Berger, 1996). In a recent experimental study, Sidelnik \& Young (2006) determined that a hand with $10^{\circ}$ of separation between fingers created more stroke force than a fingers-together configuration, across all attack angles tested.

Furthermore, $\mathrm{C}_{\mathrm{D}}$ presented the highest values for an attack angle of $90^{\circ}$ in the three hand models $(\approx 0.90$ $<\mathrm{C}_{\mathrm{D}}<1.10$ ), whereas $\mathrm{C}_{\mathrm{L}}$ resembled a sinusoidal curve across the attack angle $\left(C_{L} \approx 0.60\right)$. These results are quite similar to the ones already described with experimental methodologies (e.g., Schleihauf, 1979; Berger et al., 1995; Takagi et al., 2001).

In summary, this study showed that CFD methodology can be an important tool for coaches and swimmers to improve performance. However, the present results were obtained using steady flow simulations. Further studies should include the unsteady effects of motion, such as accelerations, decelerations, and rotations (Sanders, 1999). It would be interesting to observe whether the results would be the same as suggested by Ungerechts $\&$ Klauck (2006). These authors proposed the use of fingers slightly spread to induce flow around the hand at the beginning of the arm cycle and to create unsteady flow to allow a marked increase of propelling momentum.

Although the results of the present numerical research showed that fingers slightly spread created more force, this is a comparison of only three hand positions. In the future, there are many hand shape parameters that could be included by varying for instance wrist angle, thumb abduction, and hand configuration (flat vs. cupped palm and flexed vs. extended interphalangeal joints). 


\section{Acknowledgments}

This work was supported by the Portuguese Government by a grant of the Science and Technology Foundation (SFRH/ BD/25241/2005; PTDC/DES/098532/2008). We would like to acknowledge the staff of the Radiology Department of Hospital de São João, in Porto, Portugal, and personally to the department director, Isabel Ramos. We would also like to express our gratitude to the Med Mat Innovation Company, in Maia, Portugal, and especially to José Domingos Santos and Bruno Sá for their contributions.

\section{References}

Arellano, R., Nicoli-Terrés, J.M., \& Redondo, J.M. (2006). Fundamental hydrodynamics of swimming propulsion. Portuguese Journal of Sport Sciences, 6(Suppl. 2), 15-20.

Aritan, S., Dabnichki, P., \& Bartlett, R. (1997). Program for generation of three-dimensional finite element mesh form magnetic resonance imaging scans of human limbs. Medical Engineering \& Physics, 19(8), 681-689.

Berger, M. (1996). Force generation and efficiency in front crawl swimming. PhD Thesis. Amsterdam: Faculty of Human Movement Sciences, Vrije Universiteit.

Berger, M.A., de Groot, G., \& Hollander, A.P. (1995). Hydrodynamic drag and lift forces on human/arm models. Journal of Biomechanics, 28, 125-133.

Bixler, B., Pease, D., \& Fairhurst, F. (2007). The accuracy of computational fluid dynamics analysis of the passive drag of a male swimmer. Sports Biomechanics, 6, 81-98.

Bixler, B., \& Riewald, S. (2002). Analysis of swimmer's hand and arm in steady flow conditions using computational fluid dynamics. Journal of Biomechanics, 35, 713-717.

Candalai, R.S., \& Reddy, N.P. (1992). Stress distribution in a physical buttock model: effect of simulated bone geometry. Journal of Biomechanics, 15, 493-504.

Colwin, C. (1992). Swimming into the 21th century. Champaign, Illinois: Leisure Press.

Counsilman, J. (1968). The science of swimming. Englewood Cliffs: Prenctice-Hall Inc.

Dickinson, M.H. (2000). How animals move: an integrative view. Science, 288, 100-106.

Lauder, M.A., Dabnichki, P., \& Bartlett, R. (2001). Improved accuracy and reliability of sweepback angle, pitch angle and hand velocity calculations in swimming. Journal of Biomechanics, 34, 31-39.
Lecrivain, G., Slaouti, A., Payton, C., \& Kennedy, I. (2008). Using reverse engineering and computational fluid dynamics to investigate a lower arm amputee swimmer's performance. Journal of Biomechanics, 41, 2855-2859.

Maglischo, E.W. (2003). Swimming fastest. The essential reference on training, technique and program design. Champaign, Illinois: Human Kinetics.

Marinho, D.A., Reis, V.M., Alves, F.B., Vilas-Boas, J.P., Machado, L., Silva, A.J., et al. (2009).Hydrodynamic drag during gliding in swimming. Journal of Applied Biomechanics, 25, 253-257.

Moreira, A., Rouboa, A., Silva, A., Sousa, L., Marinho, D., Alves, F., et al. (2006). Computational analysis of the turbulent flow around a cylinder. Portuguese Journal of Sport Sciences, 6(Suppl. 1), 105.

Payton, C., Baltzopoulos, V., \& Bartlett, R. (2002). Contributions of rotations of the trunk and upper extremity to hand velocity during front crawl swimming. Journal of Applied Biomechanics, 18(3), 243-256.

Rouboa, A., Silva, A., Leal, L., Rocha, J., \& Alves, F. (2006). The effect of swimmer's hand/forearm acceleration on propulsive forces generation using computational fluid dynamics. Journal of Biomechanics, 39, 1239-1248.

Sanders, R.H. (1999). Hydrodynamic characteristics of a swimmer's hand. Journal of Applied Biomechanics, 15, 3-26.

Schleihauf, R.E. (1979). A hydrodynamic analysis of swimming propulsion. In J. Terauds \& E.W. Bedingfield (Eds.), Swimming III (pp. 70-109). Baltimore: University Park Press.

Sidelnik, N.O., \& Young, B.W. (2006). Optimising the freestyle swimming stroke: the effect of finger spread. Sports Engineering, 9, 129-135.

Silva, A.J., Rouboa, A., Moreira, A., Reis, V., Alves, F., VilasBoas, J.P., et al. (2008). Analysis of drafting effects in swimming using computational fluid dynamics. Journal of Sports Science and Medicine, 7(1), 60-66.

Takagi, H., Shimizu, Y., Kurashima, A., \& Sanders, R. (2001). Effect of thumb abduction and adduction on hydrodynamic characteristics of a model of the human hand. In J. Blackwell, \& R. Sanders (Eds.), Proceedings of Swim Sessions of the XIX International Symposium on Biomechanics in Sports (pp. 122-126). San Francisco: University of San Francisco.

Ungerechts, B., \& Klauck, J. (2006). Consequences of unsteady flow effects for functional attribution of swimming strokes. Portuguese Journal of Sport Sciences, 6(Suppl. 2), 109-111. 